16. National Institutes of Health. https://www.edi.nih.gov/people resources/advancing-racial-equity/nih-workforce-profile-fy $21 \mathrm{q} 02$ (accessed 31 August 2021).

17. Bernard, M. A. https://diversity.nih.gov/blog/2021-05-25-clo ser-look-nihs-distinguished-scholars-program-dr-carl-hashimoto (accessed 31 August 2021).

18. Beech, B. M. et al. Acad. Med. 88, 541-549 (2013).

19. Campbell, A. G. et al. CBE Life Sci. Educ. 12, 394-402 (2014).

20. Kalev, A., Dobbin, F. \& Kelly, E. Am. Sociol. Rev. 71, 589-617 (2006).
21. National Institutes of Health. https://diversity.nih.gov/programs partnerships/recruitment-search-protocol (accessed 31 August 2021).

Acknowledgements

We are grateful for the assistance provided by ICF Next in producing this manuscript, and to V. Rucker, the UNITE program manager, for her careful review and input regarding the final version of the manuscript.
Author contributions

Lead author duties were performed by M.A.B., with all authors contributing equally to the review and finalization of the manuscript.

Competing interests

The authors declare no competing interests.

\title{
Promoting diversity and inclusion in STEMM starts at the top
}

STEMM organizations must be proactive in advancing diversity, equity and inclusion.

\section{Marcia McNutt and Laura Castillo-Page}

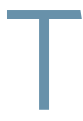
he COVID-19 pandemic offers stark evidence that without greater diversity, equity and inclusion (DEI), science is prevented from fulfilling its fundamental obligations to society. In the United States, Black/African Americans, Hispanic/Latino/ Latina/Latinx Americans, and American Indians are dying from COVID-19 at higher rates than those of other groups ${ }^{1}$. In addition, populations under-represented in science are less likely to trust in any of the several vaccines against COVID-19 that are protecting hundreds of millions of people worldwide ${ }^{2}$. The COVID-19 pandemic has also negatively impacted the careers of white women and women of color in academic science, technology, engineering, mathematics and medicine (STEMM) fields as the disruptions from the pandemic disproportionally affected their 'engagement, experience and retention ${ }^{3}$. The pandemic has thus underscored the urgency of efforts to advance DEI in science.

A more diverse, inclusive scientific workforce and culture, together with a more inclusive approach to how research is conducted, would result in more attention on addressing poorer health outcomes for racial and ethnic minority populations and other underserved communities ${ }^{4}$, more confidence within these communities that proven solutions are indeed in the best interests of all populations ${ }^{5}$, and greater overall creativity, innovation and excellence in research 6 .

The National Academy of Sciences (NAS) has recognized its own obligation in this effort. The NAS identifies and elevates

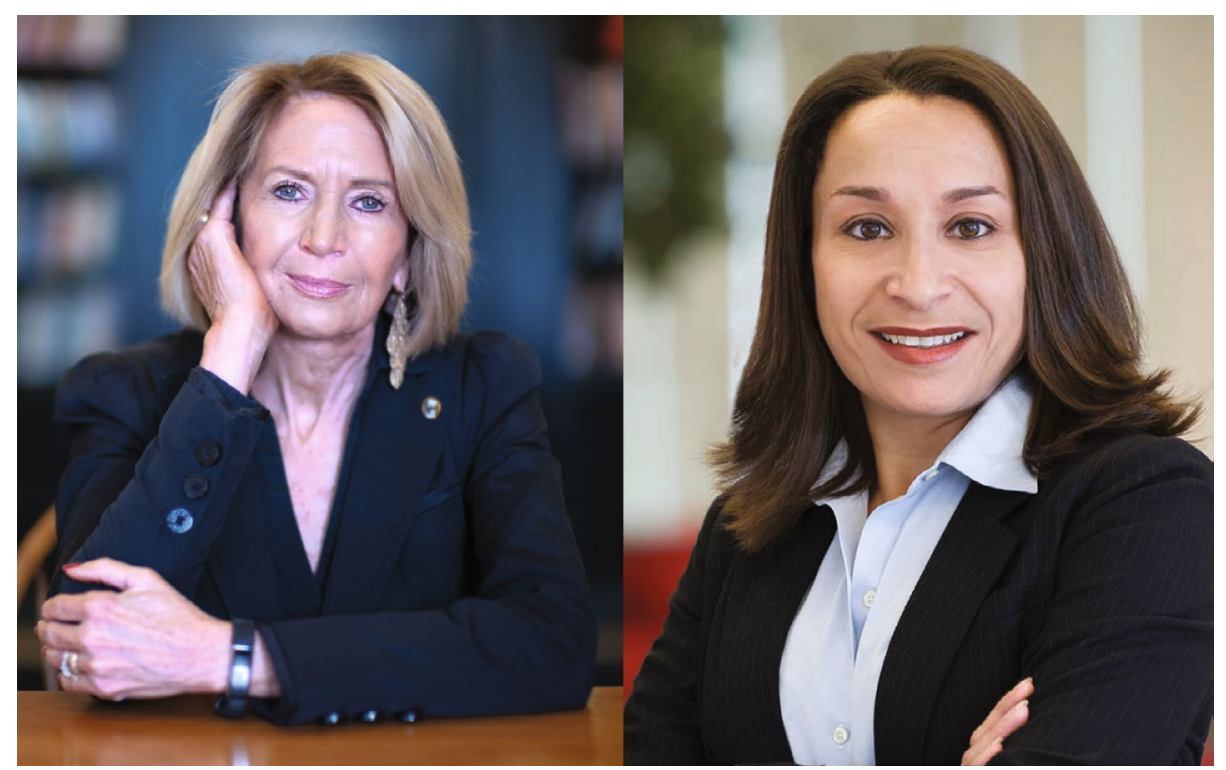

Marcia McNutt (left), credit: Chris Michel. Laura Castillo-Page (right), credit: Richard Greenhouse.

the best science in the United States and the world by electing to membership the most impactful and creative scientists. In turn, the National Academies of Sciences, Engineering, and Medicine (NASEM) calls on members from all three academies and other volunteer experts to serve on study committees that offer their best advice in addressing pressing scientific issues such as the COVID-19 pandemic and climate change. For too many decades, however, the class of NAS members elected annually and the advisory committees on which they served consisted mostly of white men, although progress has been made in recent years, especially in the number of women being elected and serving on committees.

The NAS is committed to advancing diversity both in the scientific enterprise at large and within in its own ranks. To this end, in recent years, the academy has made several reforms to the membership 
election process. The NAS Council - its senior leadership body - felt strongly that there should be no change to the criterion for election to the academy: excellence of scientific contributions. The academy designed an intentional and proactive approach to DEI that did not compromise standards but instead focused on more broadly seeking out and nominating for membership extraordinary scientists who may have been missed in the past.

The success of this approach is best illustrated in the steady progress in increasing the number of women who are elected to membership. This year, women make up almost half of the newly elected NAS members in the United States, the most ever elected in a single year. The National Academy of Engineering and the National Academy of Medicine also report similar impressive gains achieved through the use of slightly different approaches.

These changes are improving the content of the work of the NASEM while at the same time helping to advance more inclusivity within STEMM fields. Leading female NAS members have used the convening power of the NASEM to benefit all women in STEMM. For instance, the NASEM Committee on Women in Science, Engineering, and Medicine issued a landmark report on sexual harassment in academia that makes the argument that sexual harassment has not been eliminated in academic settings but has been merely driven underground ${ }^{7}$. Its recommendations for changing the culture in academia to one of inclusivity are being implemented around the nation and beyond. A follow-up report on the under-representation of women in STEMM disciplines emphasized the double burden placed on women of color and identifies evidence-based policies and procedures to increase not only gender diversity but also racial and ethnic diversity ${ }^{8}$.

Following its own advice, the NAS also adopted its first code of conduct and reversed its century-and-a-half-old policy of membership for life by changing its bylaws to allow members to be removed for severe violations. Governance changes at the National Academy of Engineering and the National Academy of Medicine now also allow membership to be revoked for violation of their codes of conduct.

Although the NAS still has a long way to go, it has also made progress in electing scientists of color. Until a few years ago, they were rarely elected to the NAS, but in 2021, nine scientists of color were elected. In addition, more researchers of color are serving in leadership positions on the NAS Council and on the councils of the other two academies. In 2019, the NASEM established the Roundtable on Black Men and Black Women in Science, Engineering, and Medicine. This roundtable's convening activities have addressed educational and financial barriers, the impact of racism and bias, and the role of mentoring in building professional pathways to support Black men and women in STEMM. And in 2021, a national summit was convened by the National Academies to address DEI and anti-racism in STEMM settings ${ }^{9}$.

The National Academies also recognize that simply reviewing and revising practices and policies aimed at advancing DEI is not enough to effect real change. To that end, this year, the NASEM established its first Office of Diversity and Inclusion, charged with leading efforts to embed DEI in its projects, activities, processes and policies. This office is paying particular attention to how issues of equity and inclusion can or should be addressed in its advisory work; in addition, it is working to increase the diversity of National Academies' members, volunteers and staff.

Not so long ago, the NAS might have naively argued that its membership could not reflect the diversity of the American public it serves until universities fixed the 'leaky pipeline' of too many women opting out of careers in scientific research almost before they begin, or until elementary and secondary schools started motivating more students of color to study STEMM disciplines and prepared them for success in college and beyond. But in 2021, it is simply not acceptable to wait for 'bottom-up' solutions.

As leaders of one of the nation's most preeminent scientific organizations, we have an obligation to enact change within the research community and within our own walls, and to ensure excellence, innovation and DEI in science. We can no longer simply say 'we cannot find diverse talent' or that the problem lies elsewhere. We must build our capacity, here at the top of the scientific enterprise, to lead DEI efforts and actively promote new approaches, practices and policies that bring about real results.

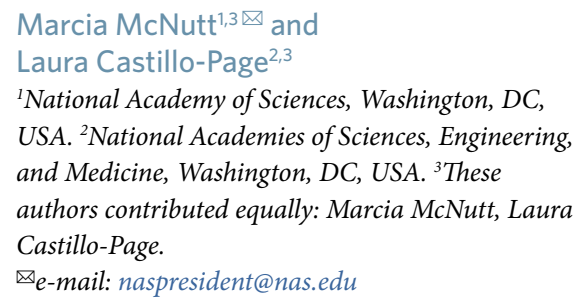

Published online: 11 November 2021 https://doi.org/10.1038/s41591-021-01496-2

References

1. Fair, M. A. et al. Science 372, 348-349 (2021).

2. Thompson, H. S. et al. JAMA Netw. Open https://doi.org/ 10.1001/jamanetworkopen.2021.11629 (2021).

3. National Academies of Sciences, Engineering, and Medicine. The National Academies Press https://doi.org/10.17226/26061 (2021).

4. Clark, U. S. et al. Nat. Hum. Behav. 4, 774-777 (2020).

5. Capers, Q. et al. J. Am. Heart Assoc. https://doi.org/10.1161/ JAHA.120.018893 (2021)

6. Swartz, T. H. et al. J. Infect. Dis. 220, S33-S41 (2019).

7. National Academies of Sciences, Engineering, and Medicine. The National Academies Press https://doi.org/10.17226/24994 (2018)

8. National Academies of Sciences, Engineering, and Medicine. The National Academies Press https://doi.org/10.17226/25585 (2020).

9. National Academies of Sciences, Engineering, and Medicine. https://www.nationalacademies.org/event/06-29-2021/ addressing-diversity-equity-inclusion-and-anti-racism-in21st-century-stemm-organizations-a-summit (2021).

Acknowledgements

We thank the leadership, members, staff and volunteers of the National Academies for their support of DEI and for helpful ideas that form the basis of this contribution.

Competing interests

The authors declare no competing interests. 